\title{
Influence of high-altitude hypoxic environments on the survival of cochlear hair cells and spiral ganglion neurons in rats
}

\author{
DONGYAN FAN ${ }^{1}$, HAILONG REN ${ }^{1}$, DUNZHU DANZENG ${ }^{1}$, HAONAN LI $^{2}$ and PING WANG ${ }^{2}$ \\ ${ }^{1}$ Department of Preventive Medicine, School of Medicine, Tibet University, Lhasa, Tibet $850002 ;{ }^{2}$ Department of \\ Otolaryngology-Head and Neck Surgery, First Hospital of Jilin University, Changchun, Jilin 130021, P.R. China
}

Received September 5, 2016; Accepted October 10, 2016

DOI: 10.3892/br.2016.791

\begin{abstract}
wAbstract. The aim of the present study was to observe the histological changes in the peripheral auditory system in rats at different time-points after relocating from low altitude to high altitude $(3,600 \mathrm{~m})$. The general physical condition of the rats was observed and cochlear tissue samples were obtained every month. The morphology and survival of the cochlear hair cells (HCs) were observed using cochlear surface preparation at 1 , $30,90,120,150$ and 180 days after moving to the plateau area. Changes in spiral ganglion neurons (SGNs) were detected at different time-points using immunofluorescence technology on frozen sections. No obvious morphological changes were observed in the cochlear HCs within 1-3 months of the rats moving to the plateau area, and there was little loss of outer $\mathrm{HCs}(\mathrm{OHCs})$ at 3 months. Cell swelling, dislocation and loss of cochlear OHCs were apparent at 4 months, and the losses of cochlear OHCs and inner HCs (IHCs) were 54 and 39\%, respectively at 6 months. The loss of SGNs was observed at 3 months, and there was a loss of $28-35 \%$ of SGNs during 3-6 months. Thus, a high-altitude hypoxic environment influenced the cochlear HCs in rats after moving to the plateau area in a time-dependent manner. The damage to SGNs occurred earlier than the HCs, although SGN damage was not aggravated with time. Furthermore, compared with cochlear HCs, cochlear SGNs were identified to be markedly more sensitive to hypoxia, and exerted an adaptive mechanism to protect neurons from hypoxia.
\end{abstract}

\section{Introduction}

Ischemia and hypoxia in the inner ear are the main factors leading to sudden deafness, acute acoustic trauma and

Correspondence to: Dr Ping Wang, Department of Otolaryngology-Head and Neck Surgery, First Hospital of Jilin University, 71 Xinmin Street, Changchun, Jilin 130021, P.R. China E-mail: drwang_ping@126.com

Key words: high-altitude hypoxia, cochlear hair cells, spiral ganglion neurons, migration presbycusis. An animal experimental study confirmed that cochlear tissues were particularly sensitive to hypoxia, and cochlear compound action potential and endocochlear potential disappeared after only $8 \mathrm{sec}$ of cochlear hypoxia (1). In addition, in vitro experiments on organ cultures of the cochlea found that hypoxia causes cochlear hair cell (HC) loss and neuronal death $(2,3)$. The mechanisms underlying hypoxia-induced inner ear damage are not clear. The Qinghai-Tibetan Plateau has high elevation and the oxygen content of the air in Lhasa is only $70 \%$ of that in the plains. In our previous study, pure tone audiometry tests were performed on youths (aged 18-25 years) living in Lhasa, and 13\% of youths living at higher elevation areas exhibited hearing loss and among those individuals, $17 \%$ were Tibetan youths, which was a greater number than Han migrants who had relocated to Tibet (4). To further study the influence of high-altitude hypoxic environment on the auditory system, cochlear samples were collected at different time-points from rats migrating to high altitude, and the morphological changes of the cochlea were observed.

\section{Materials and methods}

Experimental animals. The current study was performed in accordance with the recommendations for animal care of Tibet University (Tibet, China). The care and use of rats in the present study was approved by the Tibet University Animal Care and Use Committee.

Sixty healthy adult Wistar rats (age, 8 weeks; clean-grade), half male (weight, 190-200 g) and half female (weight, 210-220 g) were provide by Charles River Laboratories [Beijing, China; certificate of conformity, SCXK (Jing) 2012-0001]. All rats were housed (males and females separately) in a room maintained at a temperature of $20-26^{\circ} \mathrm{C}$ and a relative humidity of $45-65 \%$, with free access to water and food (complete rat pellets).

Instruments and reagents. The dissecting microscope and confocal laser scanning microscope were obtained from Olympus Corp. (Tokyo, Japan) and the freezing microtome was from Leica Microsystems GmbH (Wetzlar, Germany). Harris hematoxylin solution, 5\% goat serum, phosphate-buffered saline (PBS) and 4\% paraformaldehyde were purchased from Wuhan Boster Biological Technology, Ltd. (Wuhan, China). 
NF-200 antibody (cat.no. N0142; clone N52) and Heochst 33342 were purchased from Sigma-Aldrich (St. Louis, MO, USA) and Invitrogen Alexa 488-conjugated goat anti-mouse IgG (cat. no. A10684) was from Thermo Fisher Scientific (Waltham, MA, USA).

Experimental groups. Thirty male and 30 female rats were randomly divided into seven groups, with a control group $(n=6)$ with samples obtained from rats one day after moving to the plateau area, and six groups of samples obtained from rats $30,60,90,120,150$ and 180 days after moving to the plateau area (nine rats per group). Each included 4 or 5 male and 4 or 5 female rats. Rats were observed daily and the following were monitored: Movement, diet, drinking, defecation and hair quality and density. The rats were weighed once every three days.

Cochlear tissue collection. Rats were sacrificed by decapitation and the bilateral otocysts were removed under a dissecting microscope. The cochleae were placed in culture dishes containing $4 \%$ paraformaldehyde and the bony portion behind the otocysts was clamped using forceps. The promontorium tympani were fully exposed via opening of the otocysts via the hypotympanum. A drill hole was made in the apex of the cochlea using a 1-ml syringe needle, the bone wall at the lower edge of the round window was poked with a separating needle and the stapes were pushed through the oval window. The cochleae were perfused with $4 \%$ paraformaldehyde via the small hoe in the apical portion using the 1-ml syringe. After passing through the scala tympani and scala vestibuli, the solutions were flushed through the apex toward the base of the cochlea, and flushed out through the round and oval windows. The perfusion was performed three times. Otocysts were fixed in $4 \%$ paraformaldehyde, and placed in the refrigerator at $4^{\circ} \mathrm{C}$ for $48 \mathrm{~h}$.

Surface preparation of cochlear basilar membrane and hematoxylin staining. The fixed otocysts were then decalcified in $10 \%$ EDTA for 7 days, fixed in culture dishes containing PBS and placed under the dissecting microscope. The bony shell of the cochlea were gently dissected from the apex to the base of the cochlea under the dissection microscope, then the basilar membranes were separated from the osseous spiral lamina, which was performed from the apex toward the base of the cochlea. The basilar membranes were spread onto glass slides and the slides were immersed in absolute ethanol twice for 5 min each time, followed by immersion in $80 \%$ ethanol and distilled water for 5 and 2 min, respectively. The slides were subsequently soaked in distilled water for $5 \mathrm{~min}$ and stained with Harris hematoxylin for $5 \mathrm{~min}$. The slides were washed under running water for 1-3 sec, differentiated in $1 \%$ hydrochloric acid alcohol, fixed in gradient ethanol and mounted with glycerol.

Cochlear HC count. The surface preparation of cochlear basilar membranes were placed under the optical microscope (magnification, x400). The number of cochlear HCs was counted over $0.24-\mathrm{mm}$ intervals along the basilar membrane with the microscope eyepiece; three visual fields were selected from each basilar membrane middle turn and the mean values were taken. The hallmarks of $\mathrm{HC}$ death were considered as
A

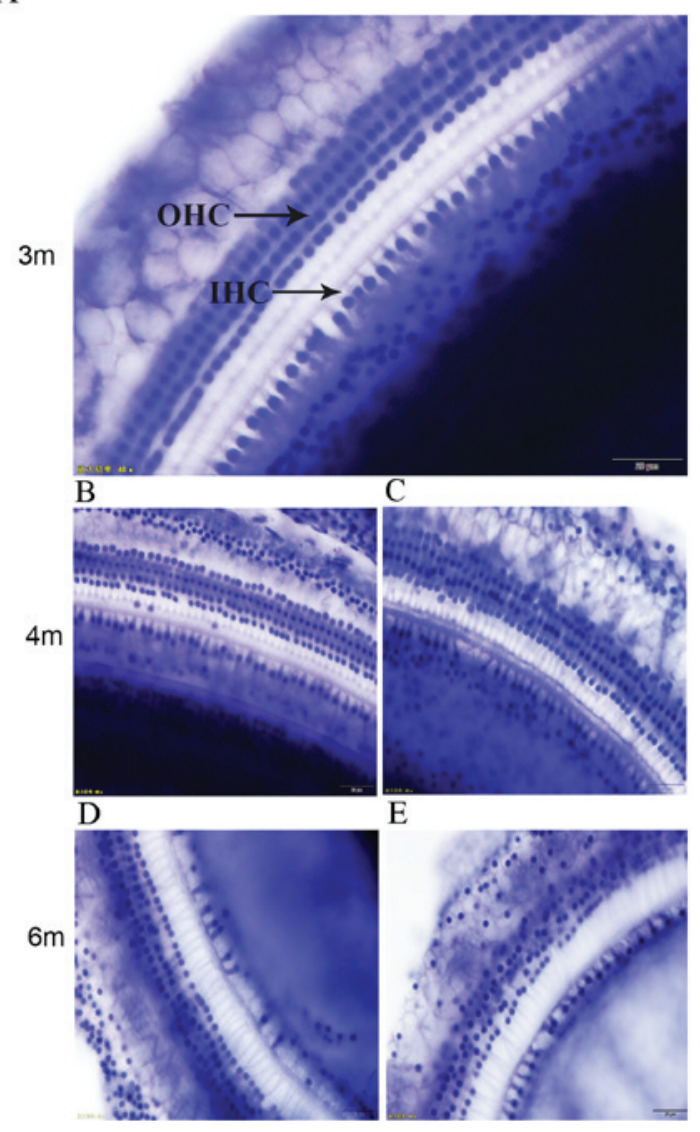

Figure 1. Morphology of cochlear Basilar Membrane at different time-points after moving to the plateau area (magnification, $x 400$; scale bar $=20 \mu \mathrm{m}$ ). (A) Cochlear basilar membrane 3 months after moving. (B and C) Cochlear basilar membrane 4 months after moving to the plateau area. (D and E) Cochlear basilar membrane 6 months after moving to the plateau area. m, months; OHC, outer hair cell; IHC, inner hair cell.

indistinct or disappearing cell boundaries and rupture or loss of cells. Visual fields at the hook and apex of the cochlea were not counted. All the data were entered into the computer by the researcher, and the distribution of $\mathrm{HC}$ loss was analyzed.

Cochlear frozen sections and immunofluorescence staining. The fixed otocysts were decalcified in 10\% EDTA solution, and the solutions were replaced every other day for a total of 15 days. The cochleae were soaked in $30 \%$ sucrose solution overnight after trimming, embedded in OCT, frozen and then sliced into sections (thickness, $10 \mu \mathrm{m}$ ) parallel to the modiolus of the cochlea. Sections were hydrated in $0.1 \mathrm{M} \mathrm{PBS}$ for $10 \mathrm{~min}$, followed by treatment with $0.1 \%$ Triton X-100 for $10 \mathrm{~min}$, and blocking with $5 \%$ goat serum for $30 \mathrm{~min}$. The sections were incubated with NF-200 antibodies (dilution, 1:400) at $4^{\circ} \mathrm{C}$ overnight, washed in PBS three times (10 min each time), then incubated with Alexa 488-conjugated goat anti-mouse IgG (dilution, 1:400) at room temperature for $60 \mathrm{~min}$, washed in PBS three times for 10 min each and mounted using glycerol. Sections were observed and photographed using a confocal microscope. After the modiolus appeared in the sections, the number of spiral ganglion neurons (SGNs) was counted in every fifth section, with a total of five sections being continuously counted under a fluorescent microscope (magnification, $x 400$ ). 
Table I. Number of cochlear HCs at different time-point after moving to the plateau area.

\begin{tabular}{lrrr}
\hline & & \multicolumn{2}{c}{ Cell count } \\
\cline { 3 - 4 } Group & Days & Outer HCs & Inner HCs \\
\hline 1 & 1 & $224.36 \pm 5.23$ & $67.44 \pm 3.43$ \\
2 & 30 & $220.47 \pm 6.18$ & $66.83 \pm 3.15$ \\
3 & 60 & $215.39 \pm 4.27$ & $66.59 \pm 2.97$ \\
4 & 90 & $210.28 \pm 4.58$ & $65.29 \pm 3.47$ \\
5 & 120 & $180.25 \pm 5.76^{\mathrm{a}}$ & $53.33 \pm 2.53^{\mathrm{a}}$ \\
6 & 150 & $161.33 \pm 4.92^{\mathrm{a}}$ & $41.24 \pm 2.86^{\mathrm{a}}$ \\
7 & 180 & $135.67 \pm 5.38^{\mathrm{a}}$ & $29.84 \pm 3.52^{\mathrm{a}}$ \\
\hline
\end{tabular}

${ }^{\mathrm{a}} \mathrm{P}<0.05$ vs. 1 day after entering into the plateau area. Values are presented as the mean \pm standard deviation. HCs, hair cells.

Table II. Number of SGNs in rats at different time-points after moving to the plateau area.

\begin{tabular}{lrc}
\hline Group & Days & \multicolumn{1}{c}{ SGNs } \\
\hline 1 & 1 & $131.47 \pm 15.36$ \\
2 & 30 & $136.94 \pm 16.58$ \\
3 & 60 & $129.31 \pm 21.28$ \\
4 & 90 & $94.32 \pm 14.16^{\mathrm{a}}$ \\
5 & 120 & $100.29 \pm 15.06^{\mathrm{a}}$ \\
6 & 150 & $90.38 \pm 8.94^{\mathrm{a}}$ \\
7 & 180 & $84.51 \pm 11.36^{\mathrm{a}}$ \\
\hline
\end{tabular}

${ }^{\mathrm{a}} \mathrm{P}<0.05$ vs. 1 day after moving to the plateau area. Values are presented as the mean \pm standard deviation. SGNs, spiral ganglion neurons.

Statistical analysis. All data are presented as the mean \pm standard deviation and were analyzed using the SPSS 17.0 statistical software (SPSS, Inc., Chicago, IL, USA). One-way analysis of variance was used and a $\mathrm{P}<0.05$ was considered to indicate a statistically significant difference.

\section{Results}

General conditions of the rats after moving to the plateau area. The vital signs and activity of rats were normal one day after moving to the plateau area, although the activity of rats was reduced, food and water intake increased for prolonged time periods under hypoxic conditions, and 15 rats succumbed between 10 and 180 days after moving to the plateau area. Monocular or binocular blindness appeared in six rats after 60 days of moving to the plateau area. The body weight of the rats continued to increase throughout the experiment, particularly in the male rats; 27 rats weighed $>500 \mathrm{~g}$ after 60 days of entering into the plateau area and nine rats weighed $>600 \mathrm{~g}$ after 180 days.
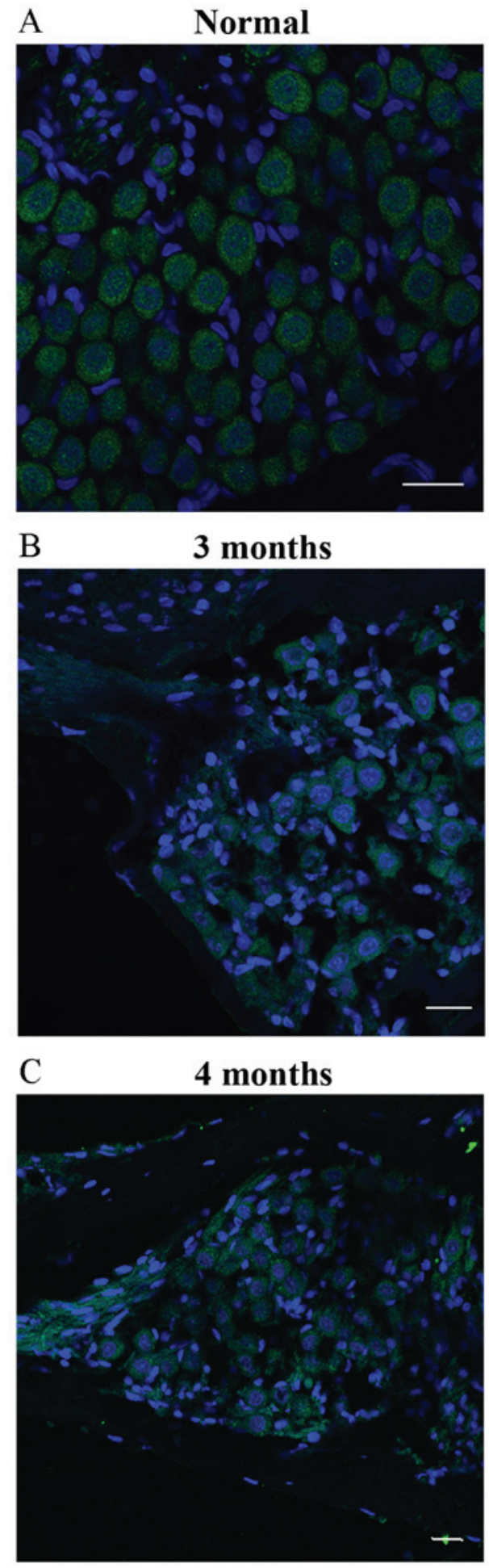

Figure 2. Morphology of SGNs at different time-points after moving to the plateau area (scale bar= $20 \mu \mathrm{m}$ ). Cochlear frozen sections were labeled with NF200 antibody, and observed and photographed using a confocal microscope. (A) Positive SGNs were indicated by green fluorescence in the normal group (magnification, $x 800$ ). (B) SGNs 3 months after moving to the plateau area (magnifications, $\mathrm{x} 600$ ). (C) SGNs 4 months after moving to the plateau area (magnification, $\mathrm{x} 400$ ). SGN, spiral ganglion neuron.

Basilar membrane changes in rat cochlea at different time-points after moving to the plateau area. The morphology of cochlear HCs in rats was normal after one day of moving to the plateau area. The HCs, which consisted of the three rows of cochlear outer $\mathrm{HCs}(\mathrm{OHCs})$ and one row of cochlear inner 
HCs (IHCs), were arranged and distributed in a uniform and regular manner, with integrated and distinct structures. No cell swelling, deformation or loss was observed.

At 1-3 months after moving to the plateau area, the arrangement and distribution of IHCs were uniform and regular with intact and distinct structures, and there were no abnormal cell changes. OHCs were arranged and distributed regularly, with only a small number of cells observed to be swollen, distorted and lost (Fig. 1A). With prolonged periods of time under hypoxia, cell swelling, dislocation, and deformation appeared in OHCs after 4-5 months; different degrees of IHC loss also began to appear (Fig. 1B and C). Six months after moving to the plateau area, the degree of HC loss was increased, cell loss was observed in the first row of OHCs, swelling and deformation of OHCs were observed, and the distance between the rows of cochlear OHCs increased. In two rats, the cochlear $\mathrm{OHC}$ boundaries became indistinct or disappeared, and the degree of cell damage was further aggravated (Fig. 1D and E).

The number of cochlear HCs on the cochlear basilar membrane were counted under a microscope at different time-points as shown in Table I.

Changes in SGNs of the cochlea in rats at different time-points after entering the plateau area. The results, obtained by immunofluorescence staining of the frozen sections in the 1 day group, showed that the NF-200-positive cells in the SGNs exhibited green fluorescence in the cell cytoplasm, which had an intact cell membrane, large, round nuclei and distinct morphology. In addition, a small quantity of glial cells and fibroblasts with small, oval or spindle-shaped nuclei surrounding the neuronal cells were observed (Fig. 2A). No significant histological changes were observed in SGNs after 1-2 months of moving to the plateau area. After 3-4 months, the number of SGNs was significantly decreased, and the number of interstitial cells was increased in the interstitial space of the ganglion (Fig. 2B and C). The number of SGNs was counted through serial sections, as shown in Table II. The number of SGNs in the 3-6 month groups was significantly decreased by $28-35 \%$ when compared with the 1 day group $(\mathrm{P}<0.01)$, no significant difference in the number of SGNs was identified between the 3-6 month group.

\section{Discussion}

In the current study cochlea HCs in rats did not demonstrate obvious changes during the first 3 months of moving to the plateau area from the low altitude area. However, morphological changes in HCs were observed with $>3$ months at high altitude. Cell swelling, deformation and dislocation were apparent in $\mathrm{OHCs}$, the space between the rows of $\mathrm{OHCs}$ widened and the cell boundaries became blurred or disappeared; certain morphological changes were subsequently found in IHCs. OHCs are the first to be damaged in a hypoxic environment, followed by the IHC as a result of prolonged hypoxic exposure. Furthermore, it was found that the degree of damage in the base of the cochlea was greater than that in the apex and the middle of the cochlea, which indicated that the cochlear damage caused by hypoxia initiates from the base of the cochlea. Cochlear blood flow is from the apex to the base of the cochlea, however, the blood flows very slowly due to the spiral structure of the cochlea; thus, in hypoxic conditions, cochlear HCs are easily damaged by insufficient blood supply to the cochlea. In addition, metabolism in the apex of the cochlea is predominantly anaerobic and the main metabolic pathway at the base of the cochlea is aerobic. Under hypoxic environments, the base of the cochlea cannot perform aerobic metabolism due to the lack of blood flow and oxygen to the brain, which causes damage to cells at the base of the cochlea $(5,6)$.

A significant loss of SGNs was observed within 3 months of moving to the plateau area, which was accompanied by the proliferation of glial cells and fibroblasts surrounding the neurons. Daniel et al (7) determined the effects of hypoxia on hearing in 10-day-old neonatal rats, and found that in adulthood, permanent hearing loss occurred with prolonged wave I-V inter-peak latencies, hypoxia-induced hearing loss may damage the central auditory pathways (8). In the current study, damage of SGNs appeared earlier than $\mathrm{HC}$ damage, and SGN damage was not aggravated over time; during 3-6 months, no significant difference was found in the number of SGNs. It has been speculated that cochlear neurons are more tolerant to hypooxia, which shows enhanced ability of survival neuron cells to adapt to the hypoxic microenvironment.

In conclusion, long term, continuous hypoxia was found to affect the survival of cochlear HCs and SGNs. Cochlear damage caused by hypoxia initiates at the base of the cochlea and spreads to the apex, with the OHCs becoming damaged first, followed by damage to the IHCs. Cochlear SGNs are markedly more sensitive to hypoxia, but SGNs are able to elicit adaptive mechanisms, which protect neurons from hypoxia. The current study presents preliminary results regarding histological changes in the peripheral auditory system of rats that migrated to a plateau area. A limitation of the present study was the lack of rat auditory function test data, due to the temporary conditions of the experiment. Further investigations are in progress that will facilitate with investigating the change of the auditory system and hypoxia adaptation process in humans that have relocated to a plateau area from a low altitude region.

\section{Acknowledgements}

The present study was supported by grants from the National Natural Science Foundation of China (grant no. 81260422).

\section{References}

1. Hess A, Bloch W, Huverstuhl J, Su J, Stennert E, Addicks K and Michel O: Expression of inducible nitric oxide synthase (iNOS/NOS II) in the cochlea of guinea pigs after intratympanical endotoxin-treatment. Brain Res 830: 113-122, 1999.

2. Olgun Y, Kırkım G, Kolatan E, Kıray M, Bağrıyanık A, Şerbetçioğlu B, Yılmaz O, Gökmen N, Ellidokuz H, Kumral A, et al: Otoprotective effect of recombinant erythropoietin in a model of newborn hypoxic-ischemic encephalopathy. Int J Pediatr Otorhinolaryngol 77: 739-746, 2013.

3. Amarjargal N, Andreeva N, Gross J, Haupt H, Fuchs J, Szczepek AJ and Mazurek B: Differential vulnerability of outer and inner hair cells during and after oxygen-glucose deprivation in organotypic cultures of newborn rats. Physiol Res 58: 895-902, 2009.

4. Ren HL, Pan YY, Gao DG, Wang G and Fan DY: Investigation on high altitude environmental effects on hearing health. Tibetan Journal of Medicine 36: 4-6, 2015. 
5. Dziennis S, Reif R, Zhi Z, Nuttall AL and Wang RK: Effects of hypoxia on cochlear blood flow in mice evaluated using Doppler optical microangiography. J Biomed Opt 17: 106003, 2012.

6. Chen W, Wang J, Chen J, Chen J and Chen Z: Relationship between changes in the cochlear blood flow and disorder of hearing function induced by blast injury in guinea pigs. Int J Clin Exp Pathol 6: 375-384, 2013.
7. Daniel SJ, McIntosh M, Akinpelu OV and Rohlicek CV: Hearing outcome of early postnatal exposure to hypoxia in Sprague-Dawley rats. J Laryngol Otol 128: 331-335, 2014.

8. Kim YJ, Kang HH, Ahn JH and Chung JW: Hypoxic changes in the central nervous system of noise-exposed mice. Acta Otolaryngol Suppl 127: 73-77, 2007. 